\title{
Image Statistics for Golden Appearance of a Painting by a Japanese Edo-era Artist Jakuchu Ito
}

\author{
Gouki Okazawa $^{1,2}$ and Hidehiko Komatsu ${ }^{1,2}$ \\ 1 Division of Sensory and Cognitive Information, \\ National Institute for Physiological Sciences, Okazaki 444-8585, Japan \\ 2 Department of Physiological Sciences,
}

Graduate University for Advanced Studies (SOKENDAI), Okazaki 444-8585, Japan

\{okazawa, komatsu\}@nips.ac.jp

\begin{abstract}
Humans use color terms to categorize huge amount of colors in the real world. Previous researches have shown that 11 basic color terms are sufficient to represent colors in many languages but recently we have found that color terms gold and silver are frequently used for images of objects with high specular reflectances. However, there are objects that appear golden but do not have clear highlights. One example can be found in Roshohakuho-zu, a painting drawn by a Japanese artist Jakuchu Ito. To find image features generating the golden appearance of this painting, we conducted psychophysical experiments using image patches extracted from the painting. We found that correlation between colors and luminances is related to goldness ratings evaluated by human subjects and manipulation of the color-luminance correlation affects the goldness of images. These results suggest that humans make use of the color-luminance correlation to perceive golden appearance of images.
\end{abstract}

Keywords: Color term gold, Color-luminance correlation, Painting.

\section{Introduction}

Color appearances of objects can be categorized with various color terms. The use of color terms is known to have common properties across languages [1]. Previous psychophysical researches in restricted experimental conditions where uniform colors are presented in isolation have shown that 11 basic color terms are sufficient to represent colors [2-4]. However, objects in the real world that have gloss, transparency, or various textures can be named with different color terms. We have recently shown that the color terms gold and silver are used for images of objects with specific ranges of chromaticities and high specular reflectances and have categorical properties comparable to the basic color terms [5]. This observation is consistent with the fact that real gold has high specular reflectance, and that the images of golden objects typically have glossy appearance. Images of objects with high specular reflectance commonly have highlights and it has been shown that highlights play important roles in gloss perception [6]. This fact

S. Tominaga, R. Schettini, and A. Trémeau (Eds.): CCIW 2013, LNCS 7786, pp. 68-79, 2013.

(C) Springer-Verlag Berlin Heidelberg 2013 
has been used in many paintings to reproduce the appearance of metallic objects including those made of gold.

However, the color term gold is used not only for object images having shiny surface with highlights but also for various non-metallic objects that do not have high specular reflectances. Impressive examples can be found in the paintings by a Japanese Edo-era artist Jakuchu Ito (1716 - 1800) named Doshoku Sai-e (Colorful Realm of Living Beingss). This masterpiece consists of 30 paintings of birds, flowers, fishes etc. drawn with a lot of colors in the traditional-style Japanese paintings where many objects appear to have gold color although they do not have any clear highlight. In Roshohakuho-zu (Pine-tree \& Phoenix, Fig. 1a), one of the paintings in this masterpiece, we can find a particularly nice example where feathers of the phoenix look golden despite the lack of specular highlights. Furthermore, any gilts or gold were not used. Instead, the painting consists of a black background paper layer and a front fabric layer made of silk colored by painting yellow pigments from the back and by painting white pigments from the front [7, 8]. These complex structures produce the golden appearance but why it occurs is not well understood. Because the painting has no clear highlight, there should be some factors causing the golden appearance instead of the highlights.

Previous psychophysical researches have shown that humans utilize various image statistics to perceive colors or surface properties. Motoyoshi et al. [9] showed that the skewness of luminance histogram correlated with glossiness of surfaces assessed by human subjects. The correlations between colors and luminances are also known to affect perception of surface properties of objects [10 14]. For example, Golz and MacLeod [10] showed that humans can dissociate colors of illumination and objects by making use of this color-luminance correlation. When the color and luminance are positively correlated (for example, more reddish parts are brighter), humans tend to assign the color to illumination (the illuminant is red). In relation to gloss perception, Nishida et al. [11] have shown that manipulation of the color of specular component affects glossy appearance indicating the importance of color-luminance relationship.

Thus, in this study we address the question about the golden appearance of Roshohakuho-zu by the image statistics approach [9]; from the painting, we examine the correlations between image statistics and goldness and manipulate image statistics to identify the important features for the golden appearance in the painting. The results suggest that the correlation between colors and luminances are the key feature for the golden appearance in Roshohakuho-zu.

\section{Experiment 1: Correlation between Image Statistics and Goldness}

We first examined the correlations between several image statistics and goldness of images to find candidate features related to the golden appearance of Roshohakuho-zu. To this end, we cropped image patches from the painting (Fig. 10) and asked human subjects to evaluate the goldness of these patches, as well as computing image statistics of them. 
(a)

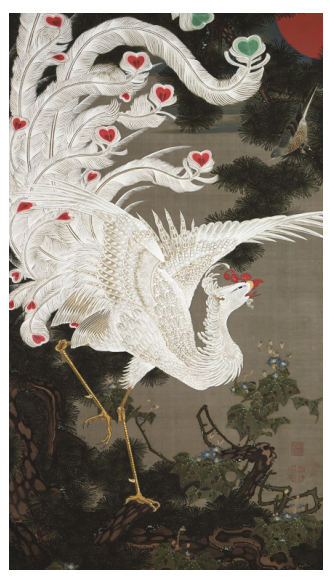

(b)

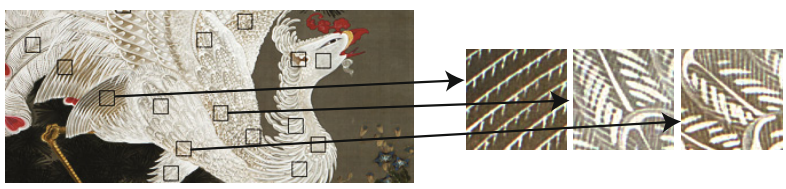

(c)
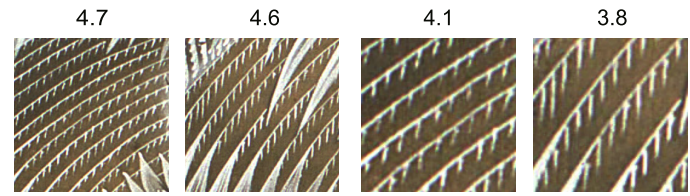

2.2

1.7

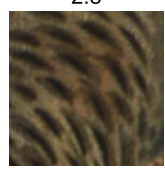

2.7

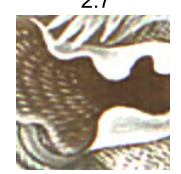

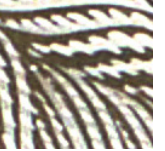

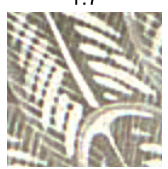

Fig. 1. The stimuli used in experiment 1. (a) Roshohakuho-zu (Pine-tree \& Phoenix) drawn by a Japanese painter Jakuchu Ito. (b) Example stimuli used in the experiments. The stimuli are image patches extracted from the painting. (c) The representative results of goldness rating evaluated by human subjects. A number above each image patch indicates the mean value of goldness rating. Values close to 1 indicate that the image did not appear golden for the subjects and values close to 5 indicate that the image appeared golden.

\subsection{Stimuli and Experimental Settings for Psychophysical Task}

We used images cropped from various parts of a digital image of Roshohakuho-zu $(4320 \times 7680$ pixels) in the experiments (Fig. 1b). We extracted 21 patches of $192 \times 192$ pixels, 12 patches of $256 \times 256$ pixels, and 8 patches of $512 \times 512$ pixels and generated in total 41 patches. Some patches contain feather regions that appear golden and others contain non-feather regions that do not appear golden. The size of all patches was rescaled to $256 \times 256$ pixels which corresponds to about 3.5 degrees of visual angle on the monitor. Color of the images was calibrated based on sRGB [15]. The range of luminances was rescaled to present on an LCD monitor used in psychophysical experiments (Totoku CCL254i2; 1 $\left.750 \mathrm{~cd} / \mathrm{m}^{2}\right)$. The background of the images was uniform black $\left(2 \mathrm{~cd} / \mathrm{m}^{2}, \mathrm{CIE}-\mathrm{x}=\right.$ 0.313 , CIE-y $=0.329$ ). Six Japanese subjects with normal or corrected-to-normal visual acuity and trichromatic color vision participated in the experiment. Four of them including the two authors have participated in similar psychophysical experiments before, while the remaining two are naïve subjects. Five of them including two naïve subjects participated in experiment 1 and all six participated in experiment 2. Each image patch was presented at the center of monitor three times for each subject. The subjects were required to press buttons to indicate whether the presented patches appeared golden or not by choosing numbers from 1 (does not appear golden) to 5 (appears golden). The stimulus was turned off 
when a button was pressed. The experiment took about $10 \mathrm{~min}$ for each subject. This research was approved by the Ethics Committee for Human Research of National Institute for Physiological Sciences.

\subsection{Results and Discussions}

Figure 17 shows the example results of goldness ratings. A number above each image patch indicates the averaged rating across the five subjects. Numbers close to 1 indicate that the patch did not appear golden and numbers close to 5 indicate that it appeared golden. As can be seen, some feather regions were rated with higher values, while other feather regions or non-feather regions were rated with lower values. Although several patches seem difficult to evaluate the goldness, the rated values were quite consistent across the subjects. The mean correlation coefficient of rated values for 41 patches between the subjects was 0.78 and the mean difference of ratings between the subjects was 0.65 . To infer the image features determining the goldness of images, we computed low-level image statistics about luminance and color of each image patch. The statistics about luminance include mean, standard deviation, and skewness of luminance histogram. With regard to color, we only considered saturation because each patch contained colors with similar hues but with varying saturations. For this reason, we computed the Euclidean distance between CIE-xy values for each pixel and the white point (D65) and used this value to calculate the statistics for colors. This value approximately corresponds to saturation of color and larger values mean saturated colors. Mean, standard deviation, and skewness of colors were computed using these distance values. In addition to these low-level statistics on luminance and color, we also considered a higher order statistics, namely, colorluminance correlation, which is suggested to be involved in color constancy and gloss perception (see Introduction). The color-luminance correlation was defined as the Pearson correlation coefficient between luminances and colors defined as the distance values. The positive correlation means that pixels with saturated colors tend to have higher luminances.

Because these statistics are mutually correlated, we computed partial correlation coefficients between the statistics and the rating of goldness averaged across all subjects (Fig. 2 a). We found that skewness of luminance histogram positively correlated with the goldness ratings $(R=0.37, P<0.03)$ whereas mean luminance $(R=-0.43, P<0.01)$ and color-luminance correlation $(R=$ $-0.52, P<0.005)$ negatively correlated with the goldness ratings. The significant correlations for mean and skewness of luminances and color-luminance correlations were also observed when we computed the single Pearson correlation coefficient. When the rated values were regressed by all statistics using the multiple regression analysis, the coefficient of determination $\left(R^{2}\right)$ became 0.79 . Figure 2 $\mathrm{b}$ shows examples of stimuli and their distributions of luminances and colors. The left example shows the image patch rated with higher value and the right one shows the patch rated with lower value. The left one has higher skewness of luminance $(0.96)$, lower mean luminance $\left(22 c d / m^{2}\right)$, and stronger 
negative correlation between luminances and CIE-x $(-0.57)$, while the right one does not (skewness $=0.36$, mean luminance $=25 \mathrm{~cd} / \mathrm{m}^{2}, R=-0.10$ ).

We also examined the effects of spatial statistics of images. We classified 41 image patches into 3 categories based on the rank of rated value in the descending order (category 1: rank 1 - 13, category 2: rank $14-27$, category 3 : rank 28 41) and computed the power spectra averaged across images in each category (Fig. 2r). There seems no particular tendency of the changes of power spectra depending on the goldness. In fact, significant differences across three categories were not observed at any spatial frequency. Thus in the current stimuli set, power spectra seem not to be related to the goldness ratings of images.

In summary, we found that three image statistics, i.e. skewness of luminances, mean luminances, and color-luminance correlations, can be considered to be candidates determining the golden appearance of Roshohakuho-zu. It has been reported that the skewness of luminance histogram of images is positively correlated with human perception of glossiness and manipulation of the skewness can actually alter perceived glossiness of image [9]. As our previous study has shown that color naming of gold is related to the glossy appearance of objects, the significant positive correlation between the goldness ratings and the skewness of luminance histogram observed in the present study may be related to the glossy appearance of image patches even though these images do not contain specular

(a)

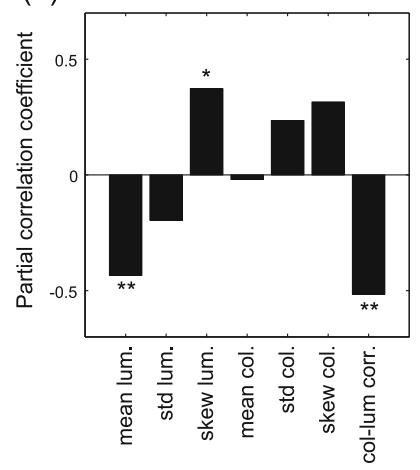

(b)

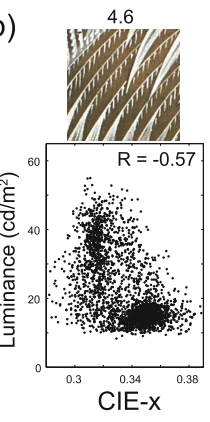

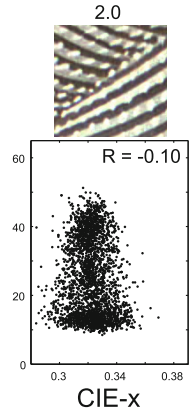

(c)

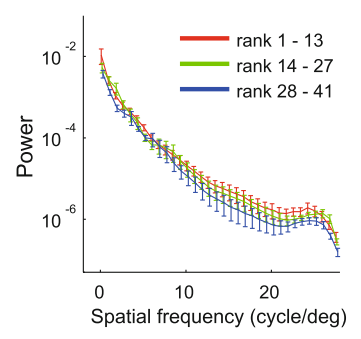

Fig. 2. The results of experiment 1. (a) Partial correlation coefficients between the rated value of goldness and image statistics. The asterisk indicates the significance of correlation; $* P<0.05, * * P<0.01$. (b) Examples of distributions of colors and luminances. A number above each image indicates the mean value of rating. Bottom panels show the scatter plots indicating the relationship between the values of CIE$\mathrm{x}$ coordinate and luminance for each pixel contained in the image. $\mathrm{R}$ indicates the correlation coefficient between CIE-x and luminance. c) Power spectrum differences between images rated with different magnitudes of rating values. All the images were classified into three categories based on the rank of rating. Each colored line indicates the average power spectrum of the images in each category. There was no significant difference across three categories at any spatial frequency. 
highlights. The significant negative correlation observed for mean luminances is rather surprising, but this might be related to a previous study, which showed that the appearance of gold deteriorated as the lightness of images increased [16]. The significantly negative correlation observed for color-luminance correlation is a new finding in this study and suggests a possibility that this is one of image statistics related to perceived goldness of images. To examine such possibility, in the subsequent experiment, we focused on this statistics and tested whether it actually affects the perceived goldness of images.

\section{Experiment 2: Manipulation of Color-Luminance Correlation Alters Goldness of Images}

In experiment 1 , we showed that the correlation between color and luminance is a candidate feature for the perceived goldness observed in Roshohakuho-zu. In experiment 2 , in order to evaluate the role of color-luminance correlation in images, we manipulated this correlation by editing image patches and evaluated the effect of the manipulation on the perceived goldness by using the same procedure conducted in experiment 1.

\subsection{Manipulation of Image Statistics}

We chose 7 samples from the 41 patches used in experiment 1 whose goldness rating values ranged from 4.6 to 1.2. To examine the effect of image statistics, we generated variants of them by manipulating chromaticities and luminances of these images (Fig. 3a). Before these manipulations, we first matched lowlevel statistics of chromaticities and luminances of all the 7 images to exclude the effects of the low-level statistics because many features or image statistics are intermingled in the image patches taken from the painting. These statistics include the luminance histogram as well as the mean and standard deviation of chromaticity coordinates in the CIE-xy chromaticity diagram. The luminance histograms of two images were matched by computing their cumulative density functions (CDF) and transforming the gray scale level of one image such that its CDFs will become identical to that of another image. The low-level image statistics of all 7 samples were matched to those of the image patch acquiring the best score for goldness rating (4.6) in experiment 1. By this operation, the effects of low-level features that exhibited significant correlations with the goldness ratings in experiment 1 , i.e. mean and skewness of luminance, are excluded in this experiment. These 7 images matched with respect to low level features are defined as "original" images, and the image labeled "original" in Fig. 3a is an example. For each of these 7 original image patches, we applied 3 manipulations, which we call "rotate 180", "color skeleton", and "col-lum skeleton", respectively (Fig. 3a).

The manipulation called "rotate 180" changes the color-luminance correlation by rotating the chromaticities of each pixel with 180 degrees around the mean chromaticity of image in the CIE-xy diagram, while the luminance of pixels 
was maintained. This operation roughly reversed the color-luminance correlation while keeping any statistics related to luminance histogram and the low level statistics of colors. The average color-luminance correlation is $-0.42 \pm 0.17$ for "original" images and $0.32 \pm 0.20$ for "rotate $180 "$ images. If color-luminance correlation is related to the perceived goldness, we can expect that the goldness ratings will change by this operation. As the original image had anisotropy in the distributions of colors on the chromaticity diagram, the "rotate 180" operation made the image to have more saturated (yellowish) pixels.

Other manipulations, i.e. "color skeleton" and "col-lum skeleton" are intended to examine the effects of color variations in images independent of the colorluminance correlation. In these manipulations, variation of color was removed using principal component analysis while maintaining the color-luminance correlation as shown in CIE-xy diagrams in Fig. 33. In "color skeleton" operation, the first and second principal components of the pixel distributions in the CIE-xy diagram were computed and the second component of each pixel was subtracted from the original chromaticity. This operation resulted in the distribution of pixels aligned with the first principal axis as shown in Fig. 33. Although the

(a)

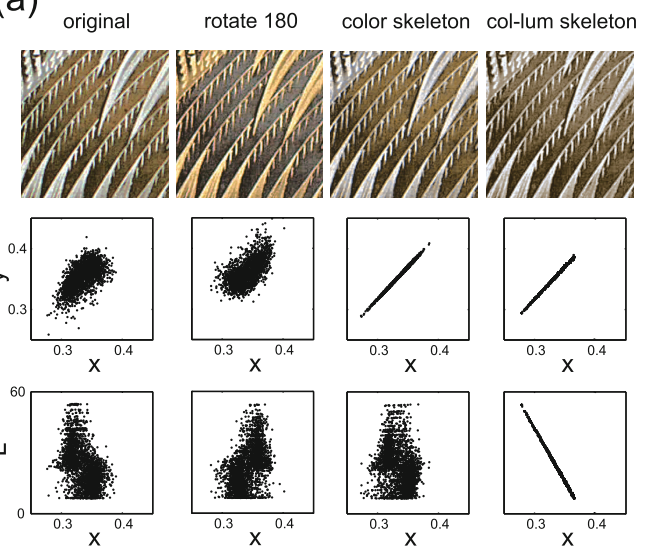

(b)

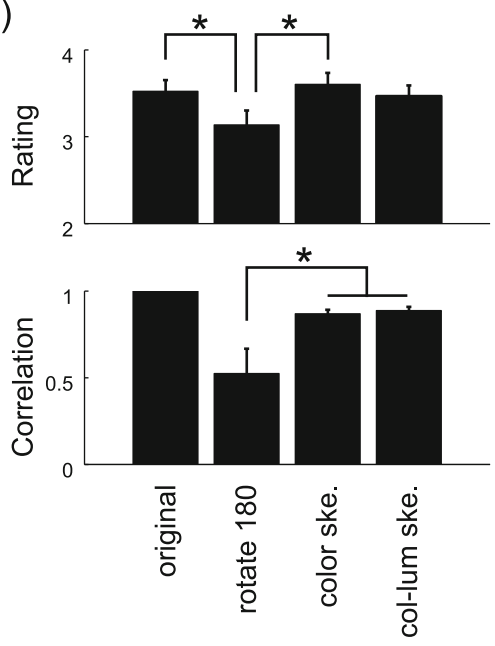

Fig. 3. The stimuli and results in experiment 2. (a) Examples of stimuli used in experiment 2 and their pixel distributions in color and luminance spaces. We selected 7 image patches from the stimuli in experiment 1 . The figure shows one of the image patches. For each patch, we manipulated images in three different ways. The images after the manipulations (top row) and their pixel distributions (middle and bottom rows) are shown. The methods of manipulations are described in the main text. (b) The upper panel shows the mean rating of goldness averaged across the 7 image patches for each manipulation. The lower panel shows the correlation coefficient of the rating of the 7 image patches between the "original" images and the manipulated images averaged across subjects. The asterisk indicates the significance of differences between different manipulations; $* P<0.05$. Error bars indicate the standard error of the mean. 
operation also slightly changed the relationship between color and luminance (compare the bottom panels of "original" and "color skeleton" in Fig. 3a), the average color-luminance correlation $(-0.42 \pm 0.17)$ did not change from "original" images. In "col-lum skeleton" manipulation, variations in the color-luminance space were further restricted by first extracting the first principal component in the CIE-xy diagram as in "color skeleton" and then computing the regression line between this first component and the luminance of pixels. The chromaticity of each pixel was shifted to locate on this regression line. We did not use the principal component method in the color-luminance space to keep the luminance of pixels unchanged. All 3 manipulations were applied for the 7 image patches, thus in total 28 images including the original ones were prepared. All images were presented three times for six Japanese subjects. Five of them participated in experiment 1 . The subjects were asked to evaluate the goldness of images as in experiment 1 . Other experimental settings were the same as in experiment 1.

\subsection{Results and Discussion}

To evaluate the effects of manipulations of image features (Fig. 3a), we first plotted the rating of goldness for each manipulation averaged across all 4 subjects and all 7 samples (Fig. 3b, upper panel). We conducted 2-way analysis of variance (ANOVA) to examine the effects of image manipulation and the effects of differences in samples. A significant main effect of image manipulation was observed $\left(F_{(3,140)}=3.35, P<0.03\right)$. The main effect of differences in samples was also significant $\left(F_{(6,140)}=15.3, P<0.001\right)$. The interaction between these two factors was not significant $\left(F_{(18,140)}=0.71, P>0.5\right)$. The posthoc multiple comparisons (Tukey-Kramer method) revealed that "color skeleton" and "rotate 180 " are significantly different $(P<0.05)$. When we compared each pair without the correction for multiple comparisons, we also observed a significant difference between "original" and "rotate 180" $(P<0.05)$, while the differences between "original" and "color skeleton" or "col-lum skeleton" were not significant (Fig. 3b, upper panel). These results indicate that color variations independent of the color-luminance correlation do not affect the goldness of images, while the color-luminance correlation affects it. One may argue that the apparent increase of saturation in "rotate 180" images has some effects on the ratings. However, because the previous reports showed that stimuli with higher saturations are more likely to be named as gold [5, 17], we consider that the apparent increase of saturation cannot explain the decrease of goldness ratings.

The significant main effect of differences in samples indicates that matching low-level statistics (the mean and standard deviation of chromaticities and the histogram of luminance) is not sufficient to match the goldness of images. In other words, there should be other critical features contributing to the differences in goldness between the samples. If the image manipulations conducted here (Fig. 3a) have disrupted these critical features, rank orders of goldness of the samples will be affected. Based on such an idea, we computed the correlation between the goldness of original 7 images and that of manipulated 7 images evaluated by the individual subjects (Figure $3 \mathrm{~b}$, lower panel). The result showed 
that the correlation coefficient dropped for "rotate $180 "(R=0.52)$, whereas it was very high for other manipulations ("color skeleton", $R=0.86$; "col-lum skeleton", $R=0.89$ ). The differences of coefficients between "rotate 180 " and "color skeleton" or "col-lum skeleton" were significant $(P<0.01$, t-test for the z values transformed from the correlation coefficient). This also supports the idea that the color-luminance correlation plays an important role in the perception of goldness.

\section{General Discussions}

Previously we have shown that images with high specular reflectance and with specific ranges of chromaticities are named as gold and the use of the color term gold has categorical properties comparable to those of the basic color terms [5]. However, feathers of phoenix in Roshohakuho-zu drawn by Jakuchu Ito appear golden although this painting does not have any clear highlight and that any materials made of gold are not used in the painting. In this study, we addressed what image features contribute to the goldness of the painting. We conducted psychophysical experiments in which subjects were required to evaluate the goldness of images that were cropped from Roshohakuho-zu and also to evaluate variants of them whose image statistics were manipulated. The results in experiment 1 showed that the skewness of luminance, mean luminance, and color-luminance correlation were correlated with the subjects' evaluations of goldness. The results in experiment 2 showed that the manipulations of color-luminance correlation disrupted the goldness of images, while removal of the color variations independent of the color-luminance correlation did not affect the perception.

Roshohakuho-zu is made of complex structures. The painting consists of a black background paper layer and a front fabric layer colored yellow from the back and colored white from the front [7, 8]. Because of the black background paper, the color of yellow on the front fabric seems to become darker [7]. This will result in the color-luminance correlation observed in this study, which may be a physical base of the golden appearance of the painting.

Several previous psychophysical researches have shown that humans utilize the color-luminance relationships to perceive surface properties of objects [10 14]. Nishida et al. [11] have shown that when the specular component of glossy object is colored red and the diffuse component is colored white, the surface looks strange and does not appear glossy. Because this situation is physically unfavorable (if the illumination was red, both the specular and diffuse components should be red), the authors speculate that humans correctly take into account the physical constraint of highlight colors. These observations seem well correspond to what occurred in our "rotate 180" manipulation in experiment 2 that made pixels with saturated (yellowish) colors brighter and pixels with unsaturated (whitish) colors darker. Although our stimuli sampled from Roshohakuho-zu does not have clear highlights and it is difficult to dissociate specular and diffuse components, some processes that automatically divides these two components and evaluates the validity of highlight colors might work in the 
human visual system. Related to this, Koida et al. 14 found that the color variations depending on the luminance cause increase in perceived brightness, which may also be related to make images having certain color-luminance correlation perceived golden. Another possible explanation of the phenomenon we found comes from the study by Golz and MacLeod [10] who have shown that humans tend to assign the color to illumination (the illuminant is red) when the color and luminance are positively correlated (for example, more reddish parts are brighter). According to this theory, it can be considered that images having negative color-luminance correlation were perceived golden because the subjects attributed the yellowish appearance of images to the color of surface and not to the color of illumination.

Other plausible explanations for the effects of color-luminance correlation on the perception can be considered from physical properties of real gold as well as multiple reflections occurring on the surface of objects made of real gold. Reflectance spectrum of metals is derived from Fresnel equation. According to Fresnel equation, reflectance spectrum of metals changes depending on the incident angle of light. When the incident angle is 0, i.e. the light is projected immediately above the surface of gold, the reflectance spectrum has a shape of high-pass curve and the reflected lights are yellowish. On the other hand, when the incident angle increase, the reflectance spectrum flattens and becomes close to the total reflection, so the reflected lights become whitish and brighter. Therefore, when lights with various incident angles are projected to the surface of gold, reflected lights contain darker yellow and brighter white, which is expected to have the color-luminance correlation. Alternatively, if the surface is not smooth but bumpy, the lights can be reflected several times on the surface before being captured by the observer's eyes. If the lights are reflected twice, the spectrum of reflected lights can be computed by multiplying the reflectance spectrum of the surface twice. Thus, if the number of reflections increases, the reflected lights become darker and saturated, which will cause the color-luminance correlation.

The results of simulation of these phenomena using the optical parameters of gold [18] are shown in Figure 4. The detail of calculations is described elsewhere (see Appendix B of [5]). In Figure 4, each dot represents the chromaticity and luminance of a reflected light with a particular angle. The colors of dots indicate the number of reflections. The right panel in Fig. 4 clearly shows that there is the color-luminance correlation derived both from the differences in incident angles and the number of reflections. Human subjects may utilize color-luminance correlation based on these kinds of physical constraints in the reflection of real gold to perceive goldness in images with such features. Future studies are required to determine which explanations listed above is more plausible.

Finally, it should be noted that some other image cues may also be used when one appreciates the real painting. The present study was conducted in a limited condition in which the subjects viewed the images presented on the display monitor. In this situation, information on the physical factors related to the image formation such as surface reflectance, fine geometrical structure and illumination environment are not available. However, when one views the real 

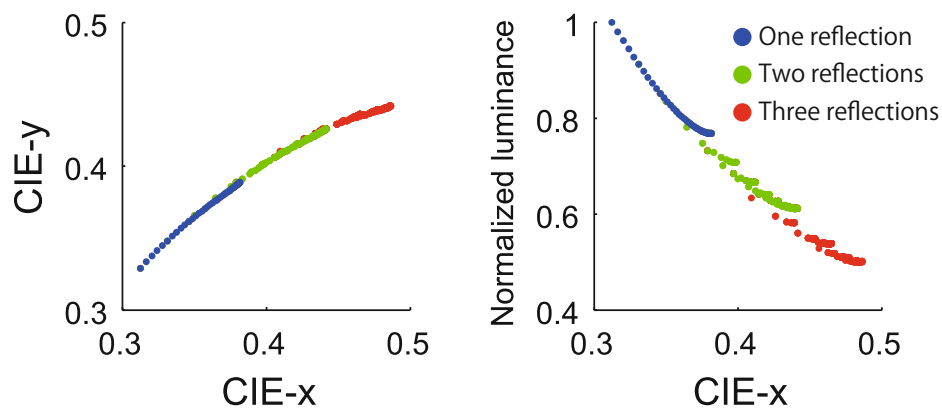

Fig. 4. Simulation of the color and luminance distributions of real gold with multiple reflections of lights with varying incident angles. Each dot represents the chromaticity and luminance of reflected light with each incident angle. Incident angles are assumed to range from 0 to 90 degrees. Colors of dots indicate the number of reflections on the surface of gold. The luminance is normalized so that the maximum luminance becomes unity.

painting, some information on the physical factors may be available, and image cues other than those considered in the present study may be used to perceive goldness of the painting.

\section{Conclusion}

In this study, we searched image features generating the golden appearance of Roshohakuho-zu, a painting by Jakuchu Ito. To this end, we extracted image patches from the painting and conducted psychophysical experiments to correlate perceived goldness and image statistics. We found that correlation between colors and luminances is a critical feature for the perception of goldness. The manipulation of color-luminance correlation actually altered goldness of images. Therefore, we conclude that the color-luminance correlation is a key feature to perceive golden appearance of objects in humans.

Acknowledgement. The color image of Roshohakuho-zu was provided by Sannomaru Shozokan, The Museum of the Imperial Collections. We thank Aya Ota for kindly allowing us to study this masterpiece. We also thank Mitsuhiro Nishiyama for giving us hint to initiate this study. This work was supported by a Grant-in-Aid for Scientific Research on Innovative Areas (No. 22135007) from the Ministry of Education, Culture, Sports, Science and Technology, Japan to H.K., and a Grant-in-Aid for JSPS Fellows from the Japan Society for the Promotion of Science to G.O. 


\section{References}

1. Berlin, B., Kay, P.: Basic Color Terms: Their Universality and Evolution. University of California Press (1969)

2. Boynton, R.M., MacLaury, R.E., Uchikawa, K.: Centroids of Color Categories Compared by Two Methods. Color Research \& Application 14, 6-15 (1989)

3. Boynton, R.M., Olson, C.X.: Locating Basic Colors in the OSA Space. Color Research \& Application 12, 94-105 (1987)

4. Uchikawa, K., Boynton, R.M.: Categorical Color Perception of Japanese Observers: Comparison with that of Americans. Vision Res. 27, 1825-1833 (1987)

5. Okazawa, G., Koida, K., Komatsu, H.: Categorical Properties of the Color Term "GOLD". J. Vis. 11(8), 1-19 (2011)

6. Beck, J., Prazdny, S.: Highlights and the Perception of Glossiness. Percept. Psychophys. 30, 407-410 (1981)

7. Ota, A.: The Exquisite Skills of Jakuchu's Depictive Expressions - After Completion of the Restoration of Doshoku Sai-e (Colorful Realm of Living Beings). Annual Report of Sannomaru Shozokan 11, 33-41 (2004) (in Japanese)

8. Hayakawa, Y., Sano, C., Miura, S.: A Scientific Examination of the Colorants Employed in All Thirty Scrolls of Ito Jakuchu's Colorful Realm of Living Beings. Annual Report of Sannomaru Shozokan 11, 42-48 (2004) (in Japanese)

9. Motoyoshi, I., Nishida, S., Sharan, L., Adelson, E.H.: Image Statistics and the Perception of Surface Qualities. Nature 447, 206-209 (2007)

10. Golz, J., MacLeod, D.I.: Influence of Scene Statistics on Colour Constancy. Nature 415, 637-640 (2002)

11. Nishida, S., Motoyoshi, I., Nakano, L., Li, Y., Sharan, L., Adelson, E.: Do Colored Highlights Look Like Highlights? [Abstract]. J. Vis. 8, 339 (2008)

12. Nakano, L., Takeuchi, T., Motoyoshi, I., Li, Y., Adelson, E., Nishida, S.: The Effect of Color Saturation and Luminance Contrast on Color Naturalness [Abstract]. J. Vis. 9, 1040 (2009)

13. Nishida, S., Motoyoshi, I., Maruya, K.: Luminance-color Interactions in Surface Gloss Perception [Abstract]. J. Vis. 11, 397 (2011)

14. Koida, K., Okazawa, G., Komatsu, H.: Clipping Illusion: a New Brightness Illusion Induced by Color Changes [Abstract in Japanese]. Vision 21, 195 (2009)

15. IEC61966-2-1/FDIS. Multimedia Systems and Equipmentt - Colour Measurement and Management - Part 2-1: Colour Management - Default RGB colour space sRGB (1999)

16. Matsumoto, T., Fukuda, K., Uchikawa, K.: Effects of Lightness on Chromaticity Regions to Yield Gold, Silver and Bronze Colors [Abstract]. J. Vis. 11, 50 (2011)

17. Matsumoto, T., Fukuda, K., Uchikawa, K.: Chromaticity and Glossiness of Gold, Silver, and Bronze Colors [Abstract]. i-Perception 2, 376-376 (2011)

18. Johnson, P.B., Christy, R.W.: Optical Constants of the Noble Metals. Physical Review B 6, 4370-4379 (1972) 